\title{
Sarcina ventriculi
}

National Cancer Institute

\section{Source}

National Cancer Institute. Sarcina ventriculi. NCI Thesaurus. Code C124384.

A species of Gram-positive cocci bacteria in the family Clostridiaceae with an oblig ate anaerobic fermentative metabolism. S. ventriculi found in soil and may be a normal part of the human gut flora. 